\title{
Comparison and uniqueness results for the periodic boundary value problem for linear first-order differential equations subject to a functional perturbation
}

\author{
Sebastián Buedo-Fernández, Daniel Cao Labora, Rosana Rodríguez-López
}

\begin{abstract}
We improve some comparison results for the periodic boundary value problem related to a first-order differential equation perturbed by a functional term. The comparison results presented cover many cases as differential equations with delay, differential equations with maxima and integro-differential equations. The interesting case of functional perturbation with piecewise constant arguments is also analyzed.
\end{abstract}

\section{Introduction}

The comparison principles are important tools for the study of the properties of the solution to differential and integral equations. In this sense, we can find many monographs devoted to the development of estimates for functions satisfying a certain differential inequality. For instance, see [1, 4, 5, 6, 7, 8]. With the help of these estimates, different techniques are applied to deduce the positivity of the solutions to differential, difference or integral equations [2] or iterative techniques in order to approximate the solutions to nonlinear differential equations [3]. Some other papers on this topic are, for instance, [12, 13, 14].

The study of comparison results for functional differential equations with piecewise constant arguments has received special attention. See [9] for first-order prob-

Rosana Rodríguez-López (corresponding author)

Departamento de Estatística, Análise Matemática e Optimización and Instituto de Matemáticas, Facultade de Matemáticas, Universidade de Santiago de Compostela, Lope Gómez de Marzoa s/n, 15782 Santiago de Compostela (Spain), e-mail: rosana.rodriguez.lopez@usc.es

Sebastián Buedo-Fernández

(same affiliation) e-mail: sebastian.buedo@usc.es

Daniel Cao Labora

(same affiliation) e-mail: daniel.cao@usc.es 
lems and $[18,26,19,20]$ for the second-order case. Some results on boundary value problems with causal operators can be found in [11].

Section 2 is devoted to present a general formulation of the problem and a key result for this work, which gives conditions to assure the existence of a nonpositive solution. In Section 3, we analyse some particular cases of the general equation, such as retarded functional differential equations, equations with minima and integrodifferential equations. In Section 4, we provide an extension of the above-mentioned result of Section 2, where the main conditions are imposed in subintervals induced by a partition of the interval where the problem is formulated. In Section 5, by bearing in mind few remarks based on the previous results, we obtain analogous results to obtain nonnegative solutions. Finally, in Section 6 we join all the conditions for nonnegativity and nonpositivity to deduce several uniqueness results.

\section{General comparison result}

Let $I=[0, T], p: L^{1}(I) \rightarrow L^{1}(I)$ and consider the problem

$$
\left\{\begin{array}{c}
v^{\prime}(t)+M v(t)+[p(v)](t)=\sigma(t), \text { a.e. } t \in I, \\
v(0)=v(T)+\lambda .
\end{array}\right.
$$

We introduce the following conditions

$$
p(w) \geq 0 \text { a.e. on } I, \text { if } w \in C(I), w \geq 0 \text { on } I,
$$

and

$$
\left\{\begin{array}{c}
\text { for all } a<b \in I \text { and } w \in C(I) \text { with } \min _{[0, b]} w \leq 0 \\
\text { we have } \int_{a}^{b}[p(w)](s) e^{M s} d s \geq \min _{s \in[0, b]}\left(w(s) e^{M s}\right)
\end{array}\right.
$$

Theorem 1 If $v \in W^{1,1}(I)$ is a solution of problem (1), $M>0, \lambda \leq 0, \sigma \leq 0$ a.e. on I and p satisfies (2) and (3), then $v \leq 0$ a.e. on I.

Proof: If $v \geq 0$ on $I$, then, using (2), we get

$$
v^{\prime}(t)=\sigma(t)-M v(t)-[p(v)](t) \leq 0
$$

for a.e. $t \in I$ and $v$ is monotonic nonincreasing. Then $v$ is a constant function since $v(0) \leq v(T)$. Let $v(t)=k$ with $k \geq 0$. Then, by (2),

$$
0 \leq M k=\sigma(t)-[p(v)](t) \leq 0, \text { a.e. } t \in I,
$$

and $v(t)=k=0$ for all $t \in I$.

This shows that either $v \equiv 0$ or there exists at least one point $t_{*} \in I$ with $v\left(t_{*}\right)<0$. If $v \leq 0$ on $I$ is not true, then there will exist $t_{0} \in I$ such that $v\left(t_{0}\right)>0$. Consider the function 


$$
z(t)=v(t) e^{M t}, t \in I .
$$

The signs of $v$ and $z$ are the same.

Then

$$
v^{\prime}(t) e^{M t}+M v(t) e^{M t} \leq-[p(v)](t) e^{M t} \text {, a.e. } t \in I,
$$

that is,

$$
z^{\prime}(t) \leq-[p(v)](t) e^{M t} \text {, a.e. } t \in I .
$$

For this function $z$, it is true that $z(0)=v(0), z\left(t_{*}\right)<0$ and $z\left(t_{0}\right)>0$. We will distinguish two cases:

Case 1: $v(0) \leq 0$.

Let $t_{1} \in\left[0, t_{0}\right)$ such that

$$
z\left(t_{1}\right)=\min _{\left[0, t_{0}\right]} z \leq 0 .
$$

Integrating (4) from $t_{1}$ to $t_{0}$ and taking into account the inequality (3), we obtain

$$
\begin{gathered}
-z\left(t_{1}\right)<z\left(t_{0}\right)-z\left(t_{1}\right) \leq-\int_{t_{1}}^{t_{0}}[p(v)](s) e^{M s} d s \\
\leq-\min _{s \in\left[0, t_{0}\right]}\left(v(s) e^{M s}\right)=-\min _{s \in\left[0, t_{0}\right]} z(s)=-z\left(t_{1}\right),
\end{gathered}
$$

that is a contradiction.

Case 2: $v(0)>0$.

Here, $z(0)>0$ and $v(T) \geq v(0)>0$, so that $z(T)>0$. Let $t_{2} \in(0, T)$ with

$$
z\left(t_{2}\right)=\min _{s \in[0, T]} z(s)<0 .
$$

Integrating (4) on $\left[t_{2}, T\right]$ and using (3) again, we get

$$
\begin{gathered}
-z\left(t_{2}\right)<z(T)-z\left(t_{2}\right) \leq-\int_{t_{2}}^{T}[p(v)](s) e^{M s} d s \\
\leq-\min _{s \in[0, T]}\left(v(s) e^{M s}\right)=-\min _{s \in[0, T]} z(s)=-z\left(t_{2}\right),
\end{gathered}
$$

which again is a contradiction.

This proves that $v \leq 0$ on $I$.

Note that condition (3) can be expressed in the following equivalent terms. Let $\widehat{w}(t)=w(t) e^{-M t}$

$$
\left\{\begin{array}{c}
\int_{a}^{b}[p(\widehat{w})](s) e^{M s} d s \geq \min _{[0, b]} w \\
\text { for all } a<b \in I \text { and } w \in C(I) \text { with } \min _{[0, b]} w \leq 0 .
\end{array}\right.
$$




\section{Particular cases}

\subsection{Retarded functional differential equations}

If

$$
[p(w)](t)=N w(\theta(t)),
$$

with $N>0, \theta: I \longrightarrow I$ such that $p: L^{1}(I) \longrightarrow L^{1}(I)$ and

$$
\theta(t) \leq t \text {, a.e. } t \in I
$$

then $p$ satisfies the hypothesis (2). Indeed, if $w \geq 0$ on $I$, then

$$
[p(w)](t)=N w(\theta(t)) \geq 0 \text { for } t \in I .
$$

If the following condition holds

$$
N \int_{0}^{T} e^{M(s-\theta(s))} d s \leq 1,
$$

then (3) is satisfied. Indeed,

$$
\begin{aligned}
& \int_{a}^{b}[p(w)](s) e^{M s} d s=\int_{a}^{b} N w(\theta(s)) e^{M s} d s \\
& =\int_{a}^{b} N w(\theta(s)) e^{M \theta(s)} e^{M(s-\theta(s))} d s \geq \min _{s \in[0, b]}\left(w(s) e^{M s}\right) N \int_{a}^{b} e^{M(s-\theta(s))} d s \\
& \geq \min _{s \in[0, b]}\left(w(s) e^{M s}\right) N \int_{0}^{T} e^{M(s-\theta(s))} d s \geq \min _{s \in[0, b]}\left(w(s) e^{M s}\right),
\end{aligned}
$$

for all $a<b \in I$ and $w \in C(I)$ with $\min w \leq 0$. Here we have used that

$$
w(\theta(t)) e^{M \theta(t)} \geq \min _{s \in[0, b]}\left(w(s) e^{M s}\right) \text {, a.e. } t \in[a, b],
$$

that is true since $\theta(t) \leq t$, a.e. $t \in I$.

Corollary 1 If $M>0, N \geq 0, \theta: I \rightarrow I, \theta(t) \leq t$, a.e. on $I$ and $v \in W^{1,1}(I)$ are such that $p: L^{1}(I) \rightarrow L^{1}(I)$ and

$$
\left\{\begin{array}{c}
v^{\prime}(t)+M v(t)+N v(\theta(t)) \leq 0, \text { a.e. } t \in I, \\
v(0) \leq v(T) \\
N \int_{0}^{T} e^{M(s-\theta(s))} d s \leq 1
\end{array}\right.
$$

then $v \leq 0$ a.e. on I.

This result improves Corollary 2 in [21]. 
An important case is $\theta(t)=[t]$, where [-] is the floor function. In this case, $p(v) \in L^{1}(I)$, for any $v \in L^{1}(I)$, and we obtain the estimate

$$
N \int_{0}^{T} e^{M(s-[s])} d s \leq 1 .
$$

If $T \leq 1$, then $[t]=0$, at least for $t \in[0, T)$ and (5) becomes

$$
N \int_{0}^{T} e^{M s} d s=\frac{N}{M}\left(e^{M T}-1\right) \leq 1 .
$$

Note that this is not a trivial case, since we can find a function $v$ with

$$
\begin{gathered}
v^{\prime}(t)+M v(t)+N v(0) \leq 0, t \in I, \\
v(0)<v(T)
\end{gathered}
$$

and $v(T)>0$. Set, for instance, $M=1, N=5, T=\frac{1}{2}$ and $v(t)=t-\frac{1}{4}$, for $t \in\left[0, \frac{1}{2}\right]$. In this case,

$$
\frac{N}{M}\left(e^{M T}-1\right)=5(\sqrt{e}-1)>1 .
$$

If $T>1$, let $k \in \mathbb{N}$, such that $k<T \leq k+1$. Then

$$
\begin{aligned}
& N \int_{0}^{T} e^{M(s-[s])} d s=N\left[\sum_{i=1}^{k} \int_{i-1}^{i} e^{M(s-i+1)} d s+\int_{k}^{T} e^{M(s-k)} d s\right] \\
& =N\left[\sum_{i=1}^{k} \frac{1}{M}\left(e^{M}-1\right)+\frac{1}{M}\left(e^{M(T-k)}-1\right)\right] \\
& =\frac{N}{M}\left[k\left(e^{M}-1\right)+\left(e^{M(T-k)}-1\right)\right] .
\end{aligned}
$$

This leads to the condition

$$
\frac{N}{M}\left[k\left(e^{M}-1\right)+e^{M(T-k)}-1\right] \leq 1 .
$$

If $k=0$, it coincides with the case $T \leq 1$. However, this estimate can be improved, as we will show below.

\subsection{Minimum case}

If $p$ satisfies the three following conditions considered in Theorem 5 of [21],

$$
p(w) \in L^{\infty}(I), \text { for every } w \in C(I)
$$




$$
\begin{gathered}
\text { ess } \inf _{t \in[0, \tau]}[p(w)](t) \geq N \min _{[0, \tau]} w, \text { for } \tau \in I \text { and } w \in C(I), \\
\frac{N}{M}\left(e^{M T}-1\right)<1,
\end{gathered}
$$

for a certain $N \geq 0$, then $p$ satisfies (2) and (3).

Indeed, let $w \in C(I), w \geq 0$ on $I$, then

$$
[p(w)](t) \geq \operatorname{ess} \inf _{s \in[0, t]}[p(w)](s) \geq N \min _{[0, t]} w \geq 0 \text {, a.e. } t \in I,
$$

and (2) holds.

Now, let $a<b \in I$ and $w \in C(I)$ with $\min _{[0, b]} w \leq 0$, then

$$
[p(w)](t) \geq \operatorname{ess} \inf _{s \in[0, t]}[p(w)](s) \geq N \min _{[0, t]} w, \text { for a.e. } t \in[a, b] .
$$

We have

$$
\begin{aligned}
& \int_{a}^{b}[p(w)](s) e^{M s} d s \geq \int_{a}^{b} N\left(\min _{[0, s]} w\right) e^{M s} d s \\
& =\int_{a}^{b} N\left(\min _{t \in[0, s]} w(t) e^{M t} e^{-M t}\right) e^{M s} d s \\
& \geq \int_{a}^{b} N\left(\min _{t \in[0, s]}\left(\min _{t \in[0, b]} w(t) e^{M t}\right) e^{-M t}\right) e^{M s} d s \\
& =\int_{a}^{b} N\left(\min _{t \in[0, b]} w(t) e^{M t}\right)\left(\max _{t \in[0, s]} e^{-M t}\right) e^{M s} d s \\
& =N \min _{t \in[0, b]}\left(w(t) e^{M t}\right) \int_{a}^{b} e^{M s} d s \\
& =N \min _{t \in[0, b]}\left(w(t) e^{M t}\right) \frac{1}{M}\left(e^{M b}-e^{M a}\right) .
\end{aligned}
$$

Also, $e^{M b}-e^{M a} \leq e^{M T}-1$ and taking into account that $\min _{[0, b]} w \leq 0$, then

$$
\int_{a}^{b}[p(w)](s) e^{M s} d s \geq \frac{N}{M}\left(e^{M T}-1\right) \min _{t \in[0, b]} w(t) e^{M t} \geq \min _{t \in[0, b]} w(t) e^{M t}
$$

and (3) is valid, even when $\frac{N}{M}\left(e^{M T}-1\right)=1$.

Thus, we have proved the following

Corollary 2 If $M>0, N \geq 0$ and $v \in W^{1,1}(I)$ are such that

$$
\left\{\begin{array}{c}
v^{\prime}(t)+M v(t)+N[p(v)](t) \leq 0, \text { a.e. } t \in I, \\
v(0) \leq v(T) \\
\frac{N}{M}\left(e^{M T}-1\right) \leq 1
\end{array}\right.
$$


where $p: L^{1}(I) \rightarrow L^{1}(I)$ satisfies (6) and (7), then $v \leq 0$ a.e. on $I$.

This result improves Theorem 5 of [21].

Note that the new result applies to functions $p: L^{1}(I) \longrightarrow L^{1}(I)$, such that

$$
[p(w)](t) \geq N \min _{[0, t]} w, \text { a.e. } t \in I,
$$

for $w \in C(I)$ and

$$
\frac{N}{M}\left(e^{M T}-1\right) \leq 1
$$

\subsection{Integral case}

Corollary 3 Let $M>0, N \geq 0$ and suppose that $p: L^{1}(I) \longrightarrow L^{1}(I)$ satisfies that

$$
[p(w)](t) \geq N \int_{0}^{t} w(s) d s, \text { a.e. } t \in I, \text { for } w \in C(I) .
$$

If $v \in W^{1,1}(I)$ is such that

$$
\left\{\begin{array}{c}
v^{\prime}(t)+M v(t)+N[p(v)](t) \leq 0, \text { a.e. } t \in I, \\
v(0) \leq v(T), \\
\frac{N}{M^{2}}\left(e^{M T}-M T-1\right) \leq 1
\end{array}\right.
$$

then $v \leq 0$ a.e. on $I$.

Proof: If $w \in C(I), w \geq 0$, then $[p(w)] \geq 0$ a.e. on $I$, so that (2) is valid. We will prove that (3) holds under the estimate

$$
\frac{N}{M^{2}}\left(e^{M T}-M T-1\right) \leq 1
$$

Indeed, for $a<b \in I$ and $w \in C(I)$ with $\min _{[0, b]} w \leq 0$, it can be proved that

$$
\begin{aligned}
& \int_{0}^{s} w(r) d r \geq \int_{0}^{s}\left(\min _{r \in[0, b]} w(r) e^{M r}\right) e^{-M r} d r \\
& =\left(\min _{r \in[0, b]} w(r) e^{M r}\right) \int_{0}^{s} e^{-M r} d r=\left(\min _{r \in[0, b]} w(r) e^{M r}\right) \frac{1-e^{-M s}}{M},
\end{aligned}
$$

for $s \in[a, b]$ and, therefore, 


$$
\begin{aligned}
& \int_{a}^{b}[p(w)](s) e^{M s} d s \geq \int_{a}^{b} N \int_{0}^{s} w(r) d r e^{M s} d s \\
& \geq \int_{a}^{b} N\left(\min _{r \in[0, b]} w(r) e^{M r}\right) \frac{1-e^{-M s}}{M} e^{M s} d s \\
& =\frac{N}{M}\left(\min _{r \in[0, b]} w(r) e^{M r}\right) \int_{a}^{b}\left(e^{M s}-1\right) d s \\
& \geq \frac{N}{M}\left(\min _{r \in[0, b]} w(r) e^{M r}\right) \int_{0}^{T}\left(e^{M s}-1\right) d s \\
& =\frac{N}{M}\left(\min _{r \in[0, b]} w(r) e^{M r}\right) \frac{e^{M T}-M T-1}{M} \\
& =\left(\min _{r \in[0, b]} w(r) e^{M r}\right) \frac{N}{M^{2}}\left(e^{M T}-M T-1\right) \geq \min _{s \in[0, b]}\left(w(s) e^{M s}\right) .
\end{aligned}
$$

This means that (3) is valid.

The estimate

$$
\frac{N}{M^{2}}\left(e^{M T}-M T-1\right) \leq 1
$$

is better than the following (that can be obtained analogously to Proposition 2 [25])

$$
\frac{N T}{M}\left(e^{M T}-1\right) \leq 1
$$

since

$$
\frac{N}{M^{2}}\left(e^{M T}-M T-1\right)<\frac{N T}{M}\left(e^{M T}-1\right) .
$$

Indeed, this is equivalent to

$$
e^{M T}-M T-1<M T\left(e^{M T}-1\right),
$$

or

$$
e^{M T}-1-M T e^{M T}<0
$$

but the function

$$
\varphi(x)=e^{x}-1-x e^{x}
$$

satisfies that $\varphi(0)=0$ and $\varphi^{\prime}(x)=-x e^{x}<0$, for $x>0$, so that $\varphi$ is nonincreasing and negative for $x>0$. Therefore, the assertion is true.

\section{Generalization of Theorem 1}

Occasionally, the equation 


$$
v^{\prime}(t)+M v(t)+[p(v)](t)=\sigma(t) \text { a.e. } t \in I,
$$

can be split into several equations

$$
v^{\prime}(t)+M v(t)+[p(v)](t)=\sigma(t), \text { a.e. } t \in\left[\alpha_{i}, \alpha_{i+1}\right],
$$

where $i=0,1, \ldots, k$ and $\left\{0=\alpha_{0}<\alpha_{1}<\cdots<\alpha_{k}<\alpha_{k+1}=T\right\}$ is a partition of $[0, T]$. This is possible, for instance, when $[p(v)](t)$ only takes into account the values of $v$ in $\left[\alpha_{i}, t\right]$, for $t \in\left[\alpha_{i}, \alpha_{i+1}\right]$ and $i=0,1, \ldots, k$. Precisely, this was the case for the delayed equation

$$
v^{\prime}(t)+M v(t)+N v([t]) \leq 0, t \in I,
$$

studied in last section. Here, if $T>1$ and $k<T \leq k+1$, we can take $\alpha_{i}=i$, for $i=0,1, \ldots, k, \alpha_{k+1}=T$ and $[p(v)](t)=N v([t])=N v(i)$, for $t \in[i, i+1)$, $i=0,1, \ldots, k-1,[p(v)](t)=N v(k)$, for $t \in[k, T]$.

In this formulation we include also the case of delayed equations with delay function $\theta$ satisfying

$$
\theta(t) \in\left[\alpha_{i}, t\right], \text { for a.e. } t \in\left[\alpha_{i}, \alpha_{i+1}\right], i=0,1, \ldots, k \text {. }
$$

Now, we will prove a result in the spirit of Theorem 1 but adapted to the property of $p$ cited above.

Theorem 2 Let $v \in W^{1,1}(I)$ be a solution of (1), $M>0, \lambda \leq 0, \sigma \leq 0$ a.e. on I and $p: L^{1}(I) \rightarrow L^{1}(I)$ such that (2) holds. Suppose that there exists $\left\{0=\alpha_{0}<\alpha_{1}<\right.$ $\left.\cdots<\alpha_{k}<\alpha_{k+1}=T\right\}$ a partition of $[0, T]$ such that $[p(v)](t)$ only depends on the values of $v$ in $\left[\alpha_{i}, t\right]$, for a.e. $t \in\left[\alpha_{i}, \alpha_{i+1}\right], i=0,1, \ldots, k$ and that

$$
\left\{\begin{array}{c}
\int_{a}^{b}[p(w)](s) e^{M s} d s \geq \min _{s \in\left[\alpha_{i}, b\right]}\left(w(s) e^{M s}\right), \quad \forall i=0,1, \ldots, k, \\
\alpha_{i} \leq a<b \leq \alpha_{i+1} \text { and } w \in C\left(\left[\alpha_{i}, \alpha_{i+1}\right]\right) \text { with } \min _{\left[\alpha_{i}, b\right]} w \leq 0 .
\end{array}\right.
$$

Then $v \leq 0$ a.e. on $I$.

Proof: We have that

$$
v^{\prime}(t)+M v(t)+[p(v)](t) \leq 0, \text { a.e. } t \in\left[\alpha_{i}, \alpha_{i+1}\right), \quad i=0,1, \ldots, k .
$$

If $v \geq 0$ on $I$, then, by (2),

$$
v^{\prime}(t) \leq-M v(t)-[p(v)](t) \leq 0
$$

for a.e. $t \in\left[\alpha_{i}, \alpha_{i+1}\right), i=0,1, \ldots, k$ and $v$ is nonincreasing on $\left[\alpha_{i}, \alpha_{i+1}\right)$, for $i=0,1, \ldots, k$. But $v$ is continuous, so that $v$ is nonincreasing on $I$ and $v$ is a constant function since $v(0) \leq v(T)$. Then $v(t)=k$ with $k \geq 0$ and

$$
0 \leq M k \leq-[p(v)](t) \leq 0, \text { a.e. } t \in I,
$$


therefore, $v(t)=k=0$, for all $t \in I$.

Now, suppose that $v\left(t_{\star}\right)<0$ for $t_{\star} \in\left[\alpha_{p}, \alpha_{p+1}\right)$ and some $p \in\{0,1, \ldots, k\}$, or $v(T)<0$.

Consider the function

$$
z(t)=v(t) e^{M t}, t \in I .
$$

Then

$$
z^{\prime}(t) \leq-[p(v)](t) e^{M t}, \text { a.e. } t \in\left[\alpha_{i}, \alpha_{i+1}\right), i=0,1, \ldots, k .
$$

If $v\left(t_{\star}\right)<0$, with $t_{\star} \in\left[\alpha_{p}, \alpha_{p+1}\right)$ and $p \in\{0,1, \ldots, k\}$, then we will prove that $v(T) \leq 0$. If $z\left(\alpha_{p+1}\right)>0$ then there exists $t_{0} \in\left[\alpha_{p}, \alpha_{p+1}\right)$ such that $z\left(t_{0}\right)=$ $\min _{\left[\alpha_{p}, \alpha_{p+1}\right]} z<0$ and integrating (10) between $t_{0}$ and $\alpha_{p+1}$ we obtain that

$$
\begin{aligned}
& -z\left(t_{0}\right)<z\left(\alpha_{p+1}\right)-z\left(t_{0}\right) \\
& \leq-\int_{t_{0}}^{\alpha_{p+1}}[p(v)](s) e^{M s} d s \leq-\min _{s \in\left[\alpha_{p}, \alpha_{p+1}\right]}\left(v(s) e^{M s}\right)=-z\left(t_{0}\right),
\end{aligned}
$$

that is absurd. Therefore, $z\left(\alpha_{p+1}\right) \leq 0$.

Now, if $p=k$, then $v(T) \leq 0$. If $p<k$, we will prove that $z\left(\alpha_{p+2}\right) \leq 0$. If $z\left(\alpha_{p+2}\right)>0$, then $t_{1} \in\left[\alpha_{p+1}, \alpha_{p+2}\right]$ is such that $z\left(t_{1}\right)=\min _{\left[\alpha_{p+1}, \alpha_{p+2}\right]} z \leq 0$ and integrating (10) between $t_{1}$ and $\alpha_{p+2}$ we get another contradiction. If $p+1=k$, we achieve $v(T) \leq 0$ and if $p+1<k$ we repeat this process until we have that $p+j=k+1, z\left(\alpha_{p+j}\right)=z(T) \leq 0$ and also $v(T) \leq 0$.

In both cases, we have that $v(T) \leq 0$. Then $z(0)=v(0) \leq v(T) \leq 0$. If there exists $t_{2} \in\left(0, \alpha_{1}\right)$ such that $z\left(t_{2}\right)>0$, then $\min _{\left[0, t_{2}\right]} z=z\left(t_{3}\right) \leq 0$. Integrating (10) for $i=0$ in $\left[t_{3}, t_{2}\right]$, we get that

$$
-z\left(t_{3}\right)<z\left(t_{2}\right)-z\left(t_{3}\right) \leq-\int_{t_{3}}^{t_{2}}[p(v)](s) e^{M s} d s \leq-\min _{s \in\left[0, t_{2}\right]}\left(v(s) e^{M s}\right)=-z\left(t_{3}\right),
$$

that is a contradiction. This implies that $z \leq 0$ on $\left[0, \alpha_{1}\right)$ and $z\left(\alpha_{1}\right) \leq 0$, since $z$ is continuous. Following an analogous procedure in the interval $\left[\alpha_{1}, \alpha_{2}\right]$, we get that $z \leq 0$ on that interval, and so on, until the interval $\left[\alpha_{k}, T\right]$, we will prove that $z \leq 0$ on $I$ and, therefore, $v \leq 0$ on $I$.

Thus, Theorem 1 is a particular case of Theorem 2, where the partition of $[0, T]$ is trivial

$$
0=\alpha_{0}<\alpha_{1}=T .
$$

Let us see how Theorem 2 improves the result obtained for the delayed differential inequality with delay function $\theta(t)=[t], t \in I$, that is 


$$
\left\{\begin{array}{c}
v^{\prime}(t)+M v(t)+N v([t]) \leq 0, \text { a.e. } t \in I \\
v(0) \leq v(T)
\end{array}\right.
$$

As we have pointed out, if $k \in \mathbb{N}, k<T \leq k+1$, we consider the partition given by $\alpha_{i}=i, i=0, \ldots, k, \alpha_{k+1}=T$. Let $i \in\{0, \ldots, k\}, \alpha_{i}=i \leq a<b \leq \alpha_{i+1}$ and $w \in C\left(\left[\alpha_{i}, \alpha_{i+1}\right]\right)$ with $\min _{\left[\alpha_{i}, b\right]} w \leq 0$. Then

$$
\begin{aligned}
& \int_{a}^{b} N w([s]) e^{M s} d s= \\
& =N \int_{a}^{b} w([s]) e^{M[s]} e^{-M[s]} e^{M s} d s \geq N \min _{s \in[i, b]}\left(w(s) e^{M s}\right) \int_{a}^{b} e^{-M[s]} e^{M s} d s \\
& =N \min _{s \in[i, b]}\left(w(s) e^{M s}\right) \int_{a}^{b} e^{M(s-i)} d s \geq N \min _{s \in[i, b]}\left(w(s) e^{M s}\right) \int_{i}^{i+1} e^{M(s-i)} d s \\
& =N \min _{s \in[i, b]}\left(w(s) e^{M s}\right) \frac{1}{M}\left(e^{M}-1\right)=\frac{N}{M}\left(e^{M}-1\right) \min _{s \in[i, b]}\left(w(s) e^{M s}\right) .
\end{aligned}
$$

If the following condition holds

$$
N \int_{i}^{i+1} e^{M(s-\theta(s))} d s \leq 1
$$

or, equivalently,

$$
\frac{N}{M}\left(e^{M}-1\right) \leq 1
$$

then

$$
\int_{a}^{b} N w([s]) e^{M s} d s \geq \min _{s \in[i, b]}\left(w(s) e^{M s}\right),
$$

for $\alpha_{i} \leq a<b<\alpha_{i+1}, i \in\{0,1, \ldots, k\}$ and (9) is valid.

We have proved the following result:

Corollary 4 If $M>0, N \geq 0, T>1$ and $v \in W^{1,1}(I)$ are such that

$$
\left\{\begin{array}{c}
v^{\prime}(t)+M v(t)+N v([t]) \leq 0, \text { a.e. } t \in I, \\
v(0) \leq v(T), \\
\frac{N}{M}\left(e^{M}-1\right) \leq 1
\end{array}\right.
$$

then $v \leq 0$ a.e. on $I$.

Compare this result with Theorem 1 [22].

In the case $T \leq 1$, the estimate $\frac{N}{M}\left(e^{M T}-1\right) \leq 1$ was obtained in Section 2 .

Corollary 5 Let $\left\{\alpha_{0}=0<\alpha_{1}<\alpha_{2}<\ldots<\alpha_{k}<\alpha_{k+1}=T\right\}$ a partition of $[0, T]$ and

$$
\theta_{i}(t):\left[\alpha_{i}, \alpha_{i+1}\right) \rightarrow\left[\alpha_{i}, \alpha_{i+1}\right), \theta_{i}(t) \leq t \text {, for a.e. } t \in\left[\alpha_{i}, \alpha_{i+1}\right) .
$$

If $p: L^{1}(I) \rightarrow L^{1}(I)$ is such that 


$$
\begin{gathered}
{[p(v)](t)=N v\left(\theta_{i}(t)\right), \text { for } t \in\left[\alpha_{i}, \alpha_{i+1}\right), i=0,1, \ldots, k-1,} \\
{[p(v)](t)=N v\left(\theta_{k}(t)\right), \text { for } t \in\left[\alpha_{k}, \alpha_{k+1}\right]}
\end{gathered}
$$

and

$$
N \int_{\alpha_{i}}^{\alpha_{i+1}} e^{M\left(s-\theta_{i}(s)\right)} d s \leq 1,
$$

for a certain $N \geq 0$, then (9) is valid. As a consequence, if $v \in W^{1,1}(I)$ is a solution of problem (1) with $M>0, \lambda \leq 0$ and $\sigma \leq 0$ a.e. on I, then $v \leq 0$ a.e. on I.

Proof: Let $i \in\{0, \ldots, k\} \alpha_{i} \leq a<b \leq \alpha_{i+1}$ and $w \in C\left(\left[\alpha_{i}, \alpha_{i+1}\right]\right)$ with $\min _{\left[\alpha_{i}, b\right]} w \leq 0$. Then, using the properties of $\theta_{i}$, we get

$$
\begin{aligned}
& \int_{a}^{b} N w\left(\theta_{i}(s)\right) e^{M s} d s=N \int_{a}^{b} w\left(\theta_{i}(s)\right) e^{M \theta_{i}(s)} e^{-M \theta_{i}(s)} e^{M s} d s \\
& \geq N \min _{s \in\left[\alpha_{i}, b\right]}\left(w(s) e^{M s}\right) \int_{a}^{b} e^{M\left(s-\theta_{i}(s)\right)} d s \\
& \geq N \min _{s \in\left[\alpha_{i}, b\right]}\left(w(s) e^{M s}\right) \int_{\alpha_{i}}^{\alpha_{i+1}} e^{M\left(s-\theta_{i}(s)\right)} d s \geq \min _{s \in\left[\alpha_{i}, b\right]}\left(w(s) e^{M s}\right) .
\end{aligned}
$$

If $\alpha_{i}=i, i=0, \ldots, k, \alpha_{k+1}=T$ and $\theta_{i}(t)=[t]=i$, for $t \in\left[\alpha_{i}, \alpha_{i+1}\right)$, then we obtain the result given in Corollary 4.

If the delay is a piecewise constant function, $\theta_{i}(t)=\alpha_{i}$, for a.e. $t \in\left[\alpha_{i}, \alpha_{i+1}\right)$, where

$$
\left\{0=\alpha_{0}<\alpha_{1}<\ldots<\alpha_{k}<\alpha_{k+1}=T\right\}
$$

then

$$
N \int_{\alpha_{i}}^{\alpha_{i+1}} e^{M\left(t-\alpha_{i}\right)} d t=\frac{N}{M}\left(e^{M\left(\alpha_{i+1}-\alpha_{i}\right)}-1\right) \leq \frac{N}{M}\left(e^{M \tau}-1\right)
$$

and we obtain the following estimate on the constants in order to guarantee the validity of (9):

$$
\frac{N}{M}\left(e^{M \tau}-1\right) \leq 1,
$$

where $\tau=\max \left\{\alpha_{i+1}-\alpha_{i}: i=0, \ldots, k\right\}$.

Corollary 6 If $p: L^{1}(I) \rightarrow L^{1}(I)$ and $\left\{0=\alpha_{0}<\alpha_{1}<\ldots<\alpha_{k}<\alpha_{k+1}=T\right\}$ are such that

$$
[p(w)](t) \geq N \min _{\left[\alpha_{i}, t\right]} w, \text { a.e. } t \in\left[\alpha_{i}, \alpha_{i+1}\right),
$$

for $i=0,1, \ldots, k, w \in C\left(\left[\alpha_{i}, \alpha_{i+1}\right]\right)$ and the estimate 


$$
\frac{N}{M}\left(e^{M \tau}-1\right) \leq 1
$$

where $\tau=\max \left\{\alpha_{i+1}-\alpha_{i}: i=0, \ldots, k\right\}$ holds, then any solution $v \in W^{1,1}(I)$ of (1) with $M>0, \lambda \leq 0, \sigma \leq 0$ a.e. on I, satisfies that $v \leq 0$ a.e. on I.

Proof: It is easy to check that (9) is true if $\frac{N}{M}\left(e^{M \tau}-1\right) \leq 1$ holds.

Corollary 7 Suppose that $p: L^{1}(I) \longrightarrow L^{1}(I)$ and

$$
\left\{0=\alpha_{0}<\alpha_{1}<\ldots<\alpha_{k}<\alpha_{k+1}=T\right\}
$$

are such that

$$
[p(w)](t) \geq N \int_{\alpha_{i}}^{t} w(s) d s \text {, a.e. } t \in\left[\alpha_{i}, \alpha_{i+1}\right),
$$

for $i=0,1, \ldots, k, w \in C\left(\left[\alpha_{i}, \alpha_{i+1}\right]\right)$ and

$$
\frac{N}{M^{2}}\left(e^{M \tau}-M \tau-1\right) \leq 1,
$$

where $\tau=\max \left\{\alpha_{i+1}-\alpha_{i}: i=0, \ldots, k\right\}$. If $v \in W^{1,1}(I)$ is a solution of $(1)$, where $M>0, \lambda \leq 0, \sigma \leq 0$ a.e. on $I$, then $v \leq 0$ a.e. on $I$.

Proof: It can be proved that (9) holds if

$$
\frac{N}{M^{2}}\left[e^{M\left(\alpha_{i+1}-\alpha_{i}\right)}-M\left(\alpha_{i+1}-\alpha_{i}\right)-1\right] \leq 1,
$$

for all $i=0,1, \ldots, k$.

However, the function $\phi(y)=e^{y}-y-1$ is nondecreasing for $y>0$, so that

$$
\phi\left(M\left(\alpha_{i+1}-\alpha_{i}\right)\right) \leq \phi(M \tau),
$$

for $i=0,1, \ldots, k$ and

$$
\frac{N}{M^{2}}\left[e^{M\left(\alpha_{i+1}-\alpha_{i}\right)}-M\left(\alpha_{i+1}-\alpha_{i}\right)-1\right] \leq \frac{N}{M^{2}}\left(e^{M \tau}-M \tau-1\right),
$$

for all $i=0,1, \ldots, k$ where the equality is valid for at least one index $i$.

\section{Nonnegativity of solutions}

Let $I=[0, T], p: L^{1}(I) \rightarrow L^{1}(I)$ and consider the problem (1) again, that is, 


$$
\left\{\begin{array}{c}
v^{\prime}(t)+M v(t)+[p(v)](t)=\sigma(t), \text { a.e. } t \in I, \\
v(0)=v(T)+\lambda .
\end{array}\right.
$$

Consider the following conditions

$$
p(w) \leq 0 \text { a.e. on } I \text {, if } w \in C(I), w \leq 0 \text { on } I
$$

and

$$
\left\{\begin{array}{c}
\text { for all } a<b \in I \text { and } w \in C(I) \text { with } \max _{[0, b]} w \geq 0, \\
\text { we have } \int_{a}^{b}[p(w)](s) e^{M s} d s \leq \max _{s \in[0, b]}\left(w(s) e^{M s}\right),
\end{array}\right.
$$

Remark 1 The former conditions can be included in the framework of Section 2 by considering $q(v)=-p(-v)$. Then, the results of this section are a direct consequence of the ones in the past sections.

Theorem 3 If $v \in W^{1,1}(I)$ is a solution of problem (1), $M>0, \lambda \geq 0, \sigma \geq 0$ a.e. on I and patisfies (11) and (12), then $v \geq 0$ a.e. on I.

Note that condition (12) can be expressed in the following way, as well.

Let $\widehat{w}(t)=w(t) e^{-M t}$

$$
\left\{\begin{array}{c}
\int_{a}^{b}[p(\widehat{w})](s) e^{M s} d s \leq \max _{[0, b]} w, \\
\text { for all } a<b \in I \text { and } w \in C(I) \text { with } \max _{[0, b]} w \geq 0 .
\end{array}\right.
$$

Next, we see some particular cases of the general framework.

\subsection{Retarded functional differential equations}

Corollary 8 Let $M>0, N \geq 0, \theta: I \rightarrow I, \theta(t) \leq t$, a.e. on $I$,

$$
[p(w)](t)=N w(\theta(t))
$$

such that $p: L^{1}(I) \longrightarrow L^{1}(I)$. If $v \in W^{1,1}(I)$ is a solution of $(1)$, where $\lambda \geq 0$, $\sigma \geq 0$ a.e. on I, then $v \geq 0$ a.e. on I.

What we have proved is that if $M>0, N \geq 0, \theta: I \rightarrow I, \theta(t) \leq t$ a.e. on $I$ and $v \in W^{1,1}(I)$ are such that $p: L^{1} \rightarrow L^{1}$ and

$$
\left\{\begin{array}{c}
v^{\prime}(t)+M v(t)+N v(\theta(t)) \geq 0, \text { a.e. } t \in I, \\
v(0) \geq v(T), \\
N \int_{0}^{T} e^{M(s-\theta(s))} d s \leq 1,
\end{array}\right.
$$

then $v \geq 0$ a.e. on $I$. 
In the case where $\theta(t)=[t], t \in[0, T]$, where $[\cdot]$ is the greatest integer function, we obtain the estimate

$$
\frac{N}{M}\left(e^{M T}-1\right) \leq 1, \text { if } T \leq 1
$$

and

$$
\frac{N}{M}\left[k\left(e^{M}-1\right)+e^{M(T-k)}-1\right] \leq 1 \text {, if } T>1 \text { and } k \in \mathbb{N} \text { such that } k<T \leq k+1 .
$$

In the last case, this is not the best estimate we can obtain.

\subsection{Maximum case}

Corollary 9 Let $M>0, \lambda \geq 0, \sigma \geq 0$ a.e. on I and $p: L^{1}(I) \longrightarrow L^{1}(I)$ such that

$$
p(w) \in L^{\infty}(I), \text { for every } w \in C(I),
$$

There exists $N \geq 0$ such that

$$
\operatorname{ess}_{\sup _{t \in[0, \tau]}}[p(w)](t) \leq N \max _{[0, \tau]} w \text {, for } \tau \in I \text { and } w \in C(I)
$$

and

$$
\frac{N}{M}\left(e^{M T}-1\right) \leq 1
$$

Then, if $v \in W^{1,1}(I)$ is a solution of $(1), v \geq 0$ a.e. on $I$.

\subsection{Integral case}

Corollary 10 Let $M>0, N \geq 0$ and suppose that $p: L^{1}(I) \longrightarrow L^{1}(I)$ satisfies that

$$
[p(w)](t) \leq N \int_{0}^{t} w(s) d s, \text { a.e. } t \in I, \text { for } w \in C(I) .
$$

If $v \in W^{1,1}(I)$ is a solution of (1), where $\lambda \geq 0, \sigma \geq 0$ a.e. on I and the estimate

$$
\frac{N}{M^{2}}\left(e^{M T}-M T-1\right) \leq 1
$$

holds, then $v \geq 0$ a.e. on I. 


\section{Uniqueness of solution}

The above results provide several uniqueness results for periodic boundary problems.

Corollary 11 Let $M>0$ and $v \in W^{1,1}(I)$ a solution to the problem

$$
\left\{\begin{array}{c}
v^{\prime}(t)+M v(t)+[p(v)](t)=0, \text { a.e. } t \in I, \\
v(0)=v(T),
\end{array}\right.
$$

where $p: L^{1}(I) \rightarrow L^{1}(I)$ is such that

$$
\begin{gathered}
p(w) \geq 0 \text { a.e. on } I, \text { if } w \in C(I), w \geq 0 \text { on } I, \\
p(w) \leq 0 \text { a.e. on } I, \text { if } w \in C(I), w \leq 0 \text { on } I, \\
\left\{\begin{array}{c}
\text { for all } a<b \in I \text { and } w \in C(I) \text { with } \min _{[0, b]} w \leq 0, \\
\text { we have } \int_{a}^{b}[p(w)](s) e^{M s} d s \geq \min _{s \in[0, b]}\left(w(s) e^{M s}\right),
\end{array}\right.
\end{gathered}
$$

and

$$
\left\{\begin{array}{l}
\text { for all } a<b \in I \text { and } w \in C(I) \text { with } \max _{[0, b]} w \geq 0, \\
\text { we have } \int_{a}^{b}[p(w)](s) e^{M s} d s \leq \max _{s \in[0, b]}\left(w(s) e^{M s}\right),
\end{array}\right.
$$

(that is, the conditions (2), (11), (3) and (12) are valid).

Then, $v \equiv 0$ a.e. on I.

Now, consider some particular cases.

Corollary 12 If $M>0, N \geq 0, \theta: I \rightarrow I, \theta(t) \leq t$, a.e. on $I$ and $v \in W^{1,1}(I)$ are such that

$$
\left\{\begin{array}{c}
v^{\prime}(t)+M v(t)+N v(\theta(t))=0, \text { a.e. } t \in I, \\
v(0)=v(T), \\
N \int_{0}^{T} e^{M(s-\theta(s))} d s \leq 1,
\end{array}\right.
$$

where the operator $[p(w)](t)=N w(\theta(t))$ satisfies that $p: L^{1}(I) \longrightarrow L^{1}(I)$, then $v \equiv 0$ a.e. on I.

Corollary 13 Let $M>0$ and $v \in W^{1,1}(I)$ a solution to the problem

$$
\left\{\begin{array}{c}
v^{\prime}(t)+M v(t)+[p(v)](t)=0, \text { a.e. } t \in I, \\
v(0)=v(T),
\end{array}\right.
$$

where $p: L^{1}(I) \rightarrow L^{1}(I)$ satisfies the three following conditions:

a) $\quad p(w) \in L^{\infty}(I)$, for $w \in C(I)$,

b) there exists $N \geq 0$, such that, for $\tau \in I$ and $w \in C(I)$

$$
\left\{\begin{array}{l}
\text { ess } \inf _{t \in[0, \tau]}[p(w)](t) \geq N \min _{[0, \tau]} w \\
\text { ess } \sup _{t \in[0, \tau]}[p(w)](t) \leq N \max _{[0, \tau]} w
\end{array}\right.
$$


c) $\quad \frac{N}{M}\left(e^{M T}-1\right) \leq 1$.

Then, $v \equiv 0$ a.e. on $I$.

Corollary 14 Let $M>0, N \geq 0$ and $v \in W^{1,1}(I)$ such that

$$
\left\{\begin{array}{c}
v^{\prime}(t)+M v(t)+N \int_{0}^{t} w(s) d s=0, \text { a.e. } t \in I, \\
v(0)=v(T),
\end{array}\right.
$$

and

$$
\frac{N}{M^{2}}\left(e^{M T}-M T-1\right) \leq 1
$$

then $v \equiv 0$ a.e. on $I$.

\section{References}

1. R. P. Agarwal and P. Y. H. Pang, Opial Inequalities with Applications in Differential and Difference Equations, Kluwer, Dordrecht, 1995.

2. R. P. Agarwal, D. O'Regan and P. J. Y. Wong, Positive Solutions of Differential, Difference and Integral Equations, Kluwer, Dordrecht, 1999.

3. G. S. Ladde, V. Lakshmikantham and A. S. Vatsala, Monotone Iterative Techniques for Nonlinear Differential Equations, Pitman, Boston, 1985.

4. V. Lakshmikantham and S. G. Leela, Differential and Integral Inequalities: Theory and Applications, Volumes I and II, Academic Press, New York, 1969.

5. B. G. Pachpatte, Inequalities for Differential and Integral Equations, Academic Press, San Diego, CA, 1998.

6. J. Schröder, Operator Inequalities, Academic Press, New York, 1980.

7. J. Szarski, Differential Inequalities, Volume 43, Monografie Matematyczne, Warsaw, 1965.

8. W. Walter, Differential and Integral Inequalities, Springer-Verlag, New York, 1970.

9. A. Cabada, J. B. Ferreiro and J. J. Nieto, Green's function and comparison principles for first order periodic differential equations with piecewise constant arguments. J. Math. Anal. Appl. 291 (2004), no. 2, 690-697.

10. Y. Chen and W. Zhuang, Monotone method for periodic boundary value problem of differential equations. J. Shandong Univ. Nat. Sci. 5 (1990) 1-7.

11. T. Jankowski, Boundary value problems with causal operators. Nonlinear Anal. 68 (2008), no. $12,3625-3632$.

12. D. Jiang and J. Wei, Monotone method for first-and second-order periodic boundary value problems and periodic solutions of functional differential equations. Nonlinear Anal. 50 (2002) 885-898.

13. E. Liz and J. J. Nieto, Periodic boundary value problems for a class of functional differential equations. J. Math. Anal. Appl. 200 (1996) 680-686.

14. E. Liz and J. J. Nieto, An abstract monotone iterative method and applications. Dynam. Systems Appl. 7 (1998) 365-375.

15. J. J. Nieto and N. Álvarez-Noriega, Periodic boundary value problems for nonlinear first order ordinary differential equations. Acta Math. Hungar. 71 (1996) 49-58.

16. J. J. Nieto, E. Liz and D. Franco, Periodic boundary value problem for nonlinear first order integro-differential equations. Integral and integro-differential equations, 237-246, Ser. Math. Anal. Appl., 2, Gordon and Breach, Amsterdam, 2000.

17. J. J. Nieto and R. Rodríguez-López, Existence and approximation of solutions for nonlinear functional differential equations with periodic boundary value conditions. Comput. Math. Appl. 40 (2000) 433-442. 
18. J. J. Nieto and R. Rodríguez-López, Green's function for second-order periodic boundary value problems with piecewise constant arguments. J. Math. Anal. Appl. 304 (2005), no. 1, 33-57.

19. J. J. Nieto and R. Rodríguez-López, Study of solutions to some functional differential equations with piecewise constant arguments. Abstr. Appl. Anal. 2012, Art. ID 851691, 25 pp.

20. J. J. Nieto and R. Rodríguez-López, Monotone method for first-order functional differential equations. Comput. Math. Appl. 52 (2006), no. 3-4, 471-484.

21. J. J. Nieto, Differential inequalities for functional perturbations of first-order ordinary differential equations. Appl. Math. Lett. 15 (2002) 173-179.

22. J. J. Nieto, A comparison result for a linear differential equation with piecewise constant delay. Glas. Mat. Ser. III, 39 (59) (2004) 73-76.

23. R. Rodríguez-López, Nonlocal boundary value problems for second-order functional differential equations. Nonlinear Anal. 74 (2011), no. 18, 7226-7239.

24. Z. Wan, Y. Chen and J. Chen, Remarks on the periodic boundary value problems for first-order differential equations. Comput. Math. Appl. 37 (1999) 49-55.

25. H. K. Xu and E. Liz, Boundary value problems for functional differential equations. Nonlinear Anal. 41 (2000) 971-988.

26. P. Yang, Y. Liu and W. Ge, Green's function for second order differential equations with piecewise constant arguments. Nonlinear Anal. 64 (2006), no. 8, 1812-1830. 